\title{
A return to cancer metabolism
}

\author{
Gregg L. Semenza
}

Received: 21 January 2011 / Accepted: 21 January 2011 / Published online: 8 February 2011

(C) Springer-Verlag 2011

\section{Introduction}

The history of science is replete with examples of important observations, which were made before their significance could be fully appreciated and which were subsequently rediscovered (or at least revisited) when additional research tools became available. The field of tumor metabolism was first explored by Otto Warburg in the early-to-mid 20th century [1]. However, interest in cancer metabolism was almost completely supplanted by the discovery of tumor suppressor genes and oncogenes in the late 20th century as the "old" approach to cancer biology (biochemistry) gave way to the "new" (molecular biology) [2]. The last several years have witnessed a dramatic resurgence of interest in the role of metabolic reprogramming in cancer progression. In this issue of the Journal of Molecular Medicine, we present five outstanding reviews [3-7] by leaders in the field of cancer metabolism, who summarize recent findings regarding key biochemical pathways and critical pathway regulators, which provide new insights into cancer progression and new targets for cancer therapy.

Chi Dang (Johns Hopkins University, Baltimore USA) and colleagues begin by discussing the highly variable nature of the metabolic profiles of normal cells and tissues [3]. These physiological profiles are then contrasted with the reprogramming of glucose metabolism that occurs in cancer cells, which is often referred to as the Warburg

G. L. Semenza $(\bowtie)$

Vascular Program, Institute for Cell Engineering;

Departments of Pediatrics, Medicine, Oncology,

Radiation Oncology, Biological Chemistry,

and the McKusick-Nathans Institute of Genetic Medicine,

The Johns Hopkins University School of Medicine,

Baltimore, MD, USA

e-mail: gsemenza@jhmi.edu phenomenon. Warburg observed that in contrast to many normal tissues, in which glucose is oxidized to $\mathrm{CO}_{2}$ and $\mathrm{H}_{2} \mathrm{O}$ under aerobic conditions but converted to lactate under hypoxic conditions, the tumors that he studied (as ex vivo slice preparations) converted large amounts of glucose to lactate even under aerobic conditions. However, the situation is complicated by the fact that, in vivo, cancers are much more hypoxic than normal tissues, and thus, lactate production may also result from the normal physiological adaptation to hypoxia. While glucose metabolism was the focus of Warburg's studies, cancer cells also take up and metabolize large amounts of glutamine. Several enzymes that play important roles in glycolysis and glutamine metabolism may represent novel targets for cancer therapy, as illustrated by recent studies from the Dang lab on inhibitors of lactate dehydrogenase [8].

Eyal Gottlieb (Beatson Institute for Cancer Research, Glasgow UK) and colleagues introduce the concept of oncometabolites, which are present at much higher levels in cancer cells than in normal cells and which promote cancer progression [4]. The tricarboxylic acid cycle enzymes succinate dehydrogenase (SDH) and fumarate hydratase (FH) have been shown to act as tumor suppressors, with loss-of-function mutations leading to the development of certain cancers. Missense mutations in the genes encoding isocitrate dehydrogenase (IDH) that alter the catalytic specificity of the enzyme have been identified in gliomas. Mutations in SDH, FH, and IDH may affect cancer progression, at least in part, by virtue of the fact that the substrate metabolites (succinate and fumarate, in the case of $\mathrm{SDH}$ and $\mathrm{FH}$ ) or product oncometabolite (2-hydroxyglutarate, in the case of mutant IDH) inhibit $\alpha$-ketoglutarate-dependent dioxygenases that regulate the activity of hypoxia-inducible factor 1, a transcription factor that plays key roles in many aspects of cancer biology including angiogenesis, metabolism, 
invasion, and metastasis [9]. Mutations in SDH, FH, or IDH may also dysregulate a second group of $\alpha$-ketoglutaratedependent dioxygenases, which function as histone demethylases. Alterations in histone methylation may mediate epigenetic changes that promote cancer progression.

Brendan Manning and Jessica Yecies (Harvard School of Public Health, Boston USA) review the role of the mammalian target of rapamycin (mTOR) as a key regulator of cell growth and proliferation whose activity is often dysregulated in cancer cells [5]. Recent studies have implicated mTOR complex 1 (mTORc1) as a metabolic regulator with effects on protein synthesis, autophagy, glucose uptake, glycolysis, mitochondrial metabolism, lipid synthesis, and the pentose phosphate pathway that is required for nucleotide and NADPH synthesis. Although mTORc1 inhibitors are in clinical trials, it is not yet clear what subset of patients will benefit from this approach, given the complex web of metabolic pathways that are impacted upon by mTORc1.

Robert Abraham (Pfizer) and colleagues further address the issues of glutamine metabolism and autophagy in cancer cells [6]. They discuss the recent exciting discovery that ammonia generated by glutaminolysis triggers autophagy and thereby promotes tumor cell survival. However, as in each of the other reviews in this series, the authors emphasize the complexity of the metabolic pathways that are involved. For example, glutamine can also suppress autophagy by stimulating mTORc1 activity, and thus, the relative activity of the glutaminolytic and mTORc1 pathways will presumably determine the extent to which autophagy is activated in any given cancer. The activity of these two pathways, in turn, will be dependent upon both genetic alterations and microenvironmental factors such as the availability of glucose, amino acids, and oxygen.

The resurgence of cancer metabolism is perhaps best appreciated by consideration of the final review in this issue [7] by Oliver Maddocks and Karen Vousden (Beatson Institute). They describe the "rebirth" of p53 as a metabolic regulator, providing a vivid illustration of the recent paradigm shift that has occurred in cancer biology, in which the characterization of tumor suppressors and oncoproteins strictly as regulators of cell proliferation and apoptosis has given way to an appreciation of their additional roles in the reprogramming tumor metabolism, which in turn is essential for cancer cell proliferation and survival.

The vast majority of advanced cancer cells take up so much more glucose than normal cells that positron emission tomography of ${ }^{18} \mathrm{~F}$-fluoro-2-deoxyglucose uptake is a highly sensitive clinical test for detecting occult metastases [10]. Could the profound metabolic differences between normal and cancer cells be exploited for treatment as well as diagnosis? The "novel" concept of targeting metabolism to kill cancer cells, which was first advanced by Warburg [1], is at risk for the kind of overblown claims that accompanied attempts to target tumor angiogenesis [11]. It is clear that much work will be required to determine the clinical contexts in which the inhibition of specific metabolic pathways may improve the efficacy of currently existing cancer therapies. The five papers in this issue of The Journal of Molecular Medicine provide a roadmap for further exploration of this "new" field of cancer metabolism.

\section{References}

1. Warburg O, Wind F, Negelein E (1927) The metabolism of tumors in the body. J Gen Physiol 8:519-530

2. McKnight SL (2010) On getting there from here. Science 330:1338-1339

3. Dang CV, Hamaker M, Sun P, Le A, Gao P (2011) Therapeutic targeting of cancer cell metabolism. J Mol Med (in press)

4. Frezza C, Pollard PJ, Gottlieb E (2011) Inborn and acquired metabolic defects in cancer. J Mol Med (in press)

5. Yecies JL, Manning BD (2011) mTOR links oncogenic signaling to tumor cell metabolism. J Mol Med (in press)

6. Shanware N, Mullen AR, DeBerardinis RJ, Abraham RT (2011) Glutamine: pleiotropic roles in tumor growth and stress resistance. $\mathrm{J}$ Mol Med (in press)

7. Maddocks ODK, Vousden KH (2011) Metabolic regulation by p53. J Mol Med (in press)

8. Le A, Cooper CR, Gouw AM, Dinavahi R, Maitra A, Deck LM, Royer RE, Vander Jagt DL, Semenza GL, Dang CV (2010) Inhibition of lactate dehydrogenase A induces oxidative stress and inhibits tumor progression. Proc Natl Acad Sci USA 107:20372042

9. Semenza GL (2010) Defining the role of hypoxia-inducible factor 1 in cancer biology and therapeutics. Oncogene 29:625-634

10. Gillies RJ, Robey I, Gatenby RA (2008) Causes and consequences of increased glucose metabolism of cancers. J Nucl Med 49:24S$42 \mathrm{~S}$

11. Marshall E (1998) The power of the front page of the New York Times. Science 280:996-997 